\title{
Three Faces of Mercaptopurine Cytotoxicity In Vitro: Methylation, Nucleotide Homeostasis, and Deoxythioguanosine in DNA ${ }^{\mathbb{S}}$
}

\author{
Sally A. Coulthard, Sarah McGarrity, Kalvin Sahota, Philip Berry, and Chris P. F. Redfern \\ Northern Institute of Cancer Research (S.A.C., S.M., P.B., C.P.F.R.) and Institute of Cellular Medicine (S.A.C., K.S.), Newcastle \\ University, Newcastle upon Tyne, United Kingdom
}

Received April 5, 2018; accepted June 1, 2018

\section{ABSTRACT}

Mercaptopurine (MP) is a cytotoxic thiopurine important for the treatment of cancer and autoimmune diseases. MP and other thiopurine drugs undergo extensive intracellular metabolism, but the mechanisms of action are poorly characterized. In particular, it is unknown how different metabolites contribute to cytotoxicity and incorporation of thiopurine bases into DNA. The aim of this study was to ask whether cytotoxicity results from the incorporation of thioguanosine nucleotides into DNA, an alternative thiopurine metabolite, or a combination of factors. Therefore, we measured the cytotoxicity, metabolism, and incorporation of thioguanosine into DNA in response to MP or MP metabolites. Thiopurine metabolites varied in cytotoxicity, with methylthioinosine-mono-phosphate and thioguanosine-tri-phosphate the most toxic, and the methyl-thioguanosine nucleotides the least. We show, using liquid chromatography-tandem mass spectrometry, how different metabolites may perturb biochemical pathways, particularly disrupting guanosine nucleotide homeostasis, that may contribute to the mechanism of action of thiopurines. Although there was no correlation between metabolite cytotoxicity and the levels of 6-methylthioinosine-monophosphate or thioguanosine incorporation into DNA as individual factors, a combined analysis suggested that these factors together had a major influence on cytotoxicity. This study emphasizes the importance of enzymes of nucleotide homeostasis, methylation, and demethylation in thiopurine effects. These results will facilitate the development of dynamic biochemical models of thiopurine biochemistry that will improve our understanding of mechanisms of action in relevant target tissues.

\section{Introduction}

The thiopurine drugs, azathioprine, mercaptopurine (MP), and 6-thioguanine have been used as immunosuppressants since the early 1950s for treatment of inflammatory bowel disease (IBD), childhood acute lymphoblastic leukemia, autoimmune hepatitis, rheumatic disease, and as a drug to reduce organ transplant rejection (Coulthard and Hogarth, 2005; Fotoohi et al., 2010). Thiopurines are prodrugs and have to be extensively metabolized to exert their cytotoxic effect. Azathioprine is non-enzymatically reduced to MP and an imidazole group. MP and 6-thioguanine undergo metabolism before exerting cytotoxicity: both drugs generate thioguanosine nucleotides via hypoxanthine guanine phosphoribosyltransferase (HPRT), but the metabolism of MP is more complex, with a different range of metabolites compared with 6-thioguanine.

Work contributing to this study was supported by a Biotechnology and Biological Sciences Research Council studentship [DTA Systems Biology], and grants from Newcastle Healthcare Charity, the JGW Patterson Foundation, and the Medical Research Council [Confidence in Concept award; Grant reference mc-pc-14101v.2].

https://doi.org/10.1124/dmd.118.081844.

S This article has supplemental material available at dmd.aspetjournals.org.
Deoxythioguanosine-tri-phosphate, a metabolite produced by MP or 6-thioguanine, is a substrate for DNA polymerases, and estimates of steady-state levels of deoxythioguanosine (dTG) in DNA of patients suggest that during replication between $0.01 \%$ and $0.1 \%$ of DNA guanines (around $10^{5}-10^{6}$ per cell) may be substituted by the thiobase (Warren et al., 1995). In addition, MP or its metabolites can be diverted from pathways of nucleotide incorporation by xanthine oxidase-mediated inactivation to thiouric acid, or by thiopurine methyltransferase (TPMT)-mediated methylation of thiopurine nucleotides or thioinosine nucleotide precursors (Coulthard and Hogarth, 2005; Fotoohi et al., 2010; Coulthard, 2012) (Fig. 1).

Several mechanisms of action have been elucidated to explain the therapeutic mechanisms of thiopurines as immunosuppressants or antileukemic drugs, and these include the inhibition of de novo purine synthesis (DNPS) (Dervieux et al., 2001; Coulthard et al., 2002), alterations in DNA methylation state via decreased levels of DNA (cytosine-5)methyltransferase 1 (DNMT1) (Coulthard et al., 2011), the disruption of guanosine-tri-phosphate (GTP) signaling (Tiede et al., 2003), and incorporation of thioguanosine nucleotides as fraudulent bases into the DNA. The latter causes DNA-protein crosslinks, single-strand breaks, interstrand crosslinks, and sister chromatid exchanges (Maybaum and Mandel, 1981, 1983; Pan and Nelson, 1990), resulting in activation of the mismatch repair

ABBREVIATIONS: DNPS, de novo purine synthesis; dTG, 6-thio-2-deoxyguanosine, deoxythioguanosine; dTG ${ }^{\mathrm{DNA}}$, deoxythioguanosine in DNA; GTP, guanosine-tri-phosphate; HPLC, high-performance liquid chromatography; meTGDP, 6-methylthioguanosine-di-phosphate; meTGMP, 6-methylthioguanosine-mono-phosphate; meTGTP, 6-methylthioguanosine-tri-phosphate; meTGXP, methyl-thioguanosine nucleoside phosphates; meTIMP, 6-methylthioinosine-mono-phosphate; meTITP, 6-methylthioinosine-tri-phosphate; MP, mercaptopurine; MTS, 3-(4,5-dimethylthiazol-2-yl)-5-(3carboxymethoxyphenyl)-2-(4-sulfophenyl)-2H-tetrazolium; TGDP, 6-thioguanosine-di-phosphate; TGMP, 6-thioguanosine-mono-phosphate; TGTP, 6-thioguanosine-tri-phosphate; TGXP, thioguanosine nucleoside phosphates; TIDP, 6-thioinosine-di-phosphate; TIMP, 6-thioinosine-mono-phosphate; TITP, 6-thioinosine-tri-phosphate. 


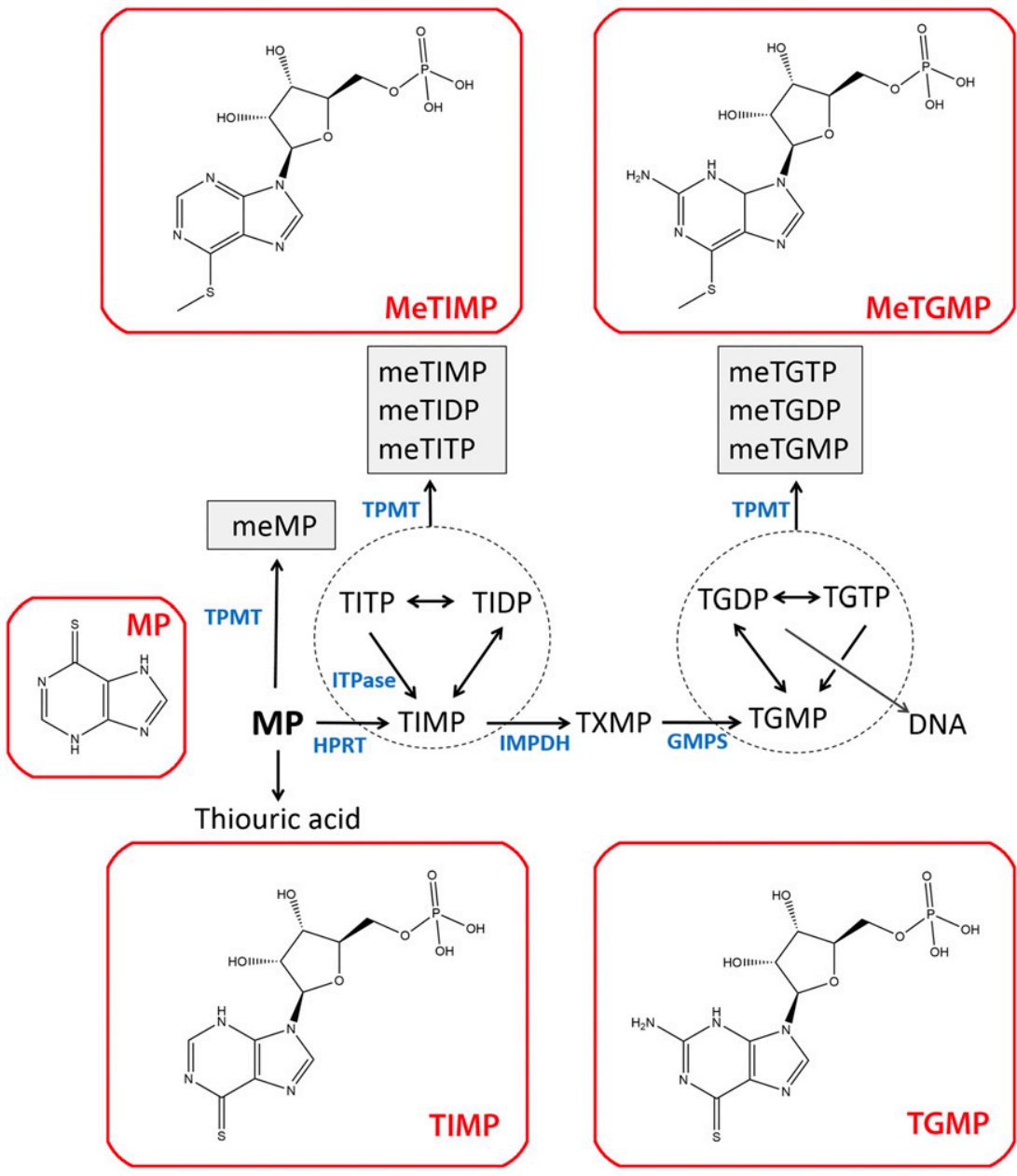

Fig. 1. Diagrammatic summary (center) of pathways of thiopurine metabolism. Thionucleotide abbreviations are as used in the text; MeMP, 6-methylmercaptopurine. Structures of thiopurine nucleoside mono-phosphates and the parent drug are shown in red boxes. GMPS, Guanosine monophosphate synthetase EC 6.3.5.2; HPRT, hypoxanthine-guanine phosphoribosyltransferase EC 2.4.2.8; IMPDH, inosine monophosphate dehydrogenase EC 1.1.1.205; ITPase, inosine triphosphate pyrophosphatase EC 3.6.1.9.

system (Swann et al., 1996) and induction of apoptosis. Furthermore, the inhibition of Rac1 activation by the binding of TGTP instead of GTP to Rac1 induces apoptosis via a mitochondrial pathway (Tiede et al., 2003).

Although therapeutic responses in leukemia correlate with levels of dTG incorporation in target cells (Ebbesen et al., 2013), the relationships between clinical outcome and other potential mechanisms of response such as Rac1 inhibition or DPNS are unknown, particularly for MP (or its precursor drug azathioprine), which has greater metabolic complexity than 6-thioguanine. Furthermore, in the context of immunosuppression, the amount of dTG in DNA does not appear to be a biologic marker of clinical response in IBD (Coulthard et al., 2017). Key issues for understanding the therapeutic mechanisms of MP are: the contribution of different metabolites and their rates of production to cytotoxicity and the incorporation of dTG into DNA, and whether these are separable consequences of different pathways of MP metabolism. These issues need to be addressed in an isogenic background to avoid cell-specific variation in expression of enzymes or other cellular characteristics that would otherwise confound interpretation. MP is used to treat childhood acute lymphoblastic leukemia as part of maintenance therapy; therefore, we employed MOLT-4 cells, a T-cell acute lymphoblastic leukemia cell line, as an experimental model. For this proof-of-principle study we developed a new liquid chromatography-mass spectrometry (LS-MS/MS) method to investigate the metabolism of MP and its metabolites, and to test the link between cytotoxicity, thiopurine metabolites, and incorporation of fraudulent bases into DNA.

\section{Materials and Methods}

Cell Culture. MOLT-4 (T-cell acute lymphoblastic leukemia cells, derived from authenticated stocks in this institute) were maintained in RPMI 1640 (Sigma-Aldrich, Poole, UK) containing 10\% fetal calf serum (SigmaAldrich) at $37^{\circ} \mathrm{C}, 5 \% \mathrm{CO}_{2}$, and were tested for mycoplasma infection every 2 months. For viability assays, repeated on three separate occasions, cells were seeded into 96 -well plates at $2 \times 10^{3} / 100 \mu \mathrm{l}$ and after 24 hours, serially diluted thiopurine metabolites (a single metabolite per plate) were added to the cells; cell viability was determined after 72 hours using the MTS assay (CellTiter $96 \mathrm{AQ}_{\text {ueous }}$ Assay; Promega, Southampton, UK) according to the manufacturer's protocol.

Twelve thiopurine metabolites were commercially available (Jena Bioscience, Jena, Germany): 6-thioguanosine-mono-phosphate (TGMP), 6-thioguanosine-diphosphate (TGDP), 6-thioguanosine-tri-phosphate (TGTP), 6-methylthioguanosinemono-phosphate (meTGMP), 6-methylthioguanosine-di-phosphate (meTGDP), 6-methylthioguanosine-tri-phosphate (meTGTP), 6-methylthioinosinemono-phosphate (meTIMP), 6-methylthioinosine-di-phosphate (meTIDP), 6-methylthioinosine-tri-phosphate (meTITP), 6-thioinosine-mono-phosphate (TIMP), 6-thioinosine-di-phosphate (TIDP) and 6-thioinosine-tri-phosphate (TITP). IUPAC names are given in Supplemental Information. For drugsensitivity assays, cells were exposed to each thiopurine metabolite individually on three separate occasions for 72 hours (three-doubling times); this is the time required for incorporation of thioguanosine nucleotides into the DNA of replicating cells and the minimum exposure time to elicit cell death by thiopurine drugs at the $\mathrm{EC}_{50}$ concentrations determined by the MTS assay. The concentrations of MP used were similar to plasma concentrations $(1.3 \mu \mathrm{M}$, range $0.75-2.2 \mu \mathrm{M})$ in children with acute lymphoblastic leukemia treated with standard doses of MP $\left(100 \mathrm{mg} / \mathrm{m}^{2}\right)$ (Estlin, 2001). 
Cell pellets for LC-MS/MS assays were generated by seeding MOLT- 4 cells at a density of $1.5 \times 10^{5} / \mathrm{ml}$ (total of $1.5 \times 10^{6}$ ) and thiopurine metabolites added to the appropriate concentrations. After 72 hours, cells were pelleted by centrifugation, resuspended, and washed twice in PBS to remove free external drug or metabolites, and washed cell pellets stored at $-80^{\circ} \mathrm{C}$ prior to extraction for analysis by LC-MS/MS. Pellets were generated for all metabolite-treated and control (no metabolite) cells in three independent experiments.

Sample Preparation for LC-MS/MS Assay of Deoxythioguanosine in DNA. Cell pellets were resuspended in $350 \mu \mathrm{l}$ of buffer B ( $400 \mathrm{mM}$ Tris, $60 \mathrm{mM}$ EDTA, $150 \mathrm{mM} \mathrm{NaCl}$ ) and $90 \mu \mathrm{l}$ of $5 \mathrm{M}$ sodium perchlorate. Samples were mixed at room temperature for 10 minutes, or until the pellet was completely dissolved, after which they were incubated at $65^{\circ} \mathrm{C}$ for 45 minutes. An equal volume of chloroform was added to each sample and mixed for 20 minutes at room temperature, followed by centrifugation at $432 \mathrm{~g}$ for 10 minutes at $4{ }^{\circ} \mathrm{C}$. The top layer was then removed and DNA precipitated by addition of 2.5 volumes of ethanol, and samples centrifuged at $16,000 \mathrm{~g}$. Precipitated DNA was resuspended in $5 \mathrm{mM}$ of Tris- $\mathrm{HCl}, \mathrm{pH} 8$, by incubation overnight at $50^{\circ} \mathrm{C}$.

Analysis of deoxythioguanosine in DNA $\left(\mathrm{dTG}^{\mathrm{DNA}}\right)$ used a previously published method (Coulthard et al., 2016). In outline, DNA was diluted to 0.2 $\mu \mathrm{g} / \mathrm{ml}$ in a total volume of $100 \mu \mathrm{l}$ of $1 \mu \mathrm{M}$ 6-(Methylthio)-9H-purine-d3 and denatured by heating to $100^{\circ} \mathrm{C}$ for 5 minutes. After being chilled on ice for 2 minutes, $10 \mu \mathrm{l}$ of $10 \times$ digestion buffer $\left(500 \mathrm{mM}\right.$ sodium acetate, $10 \mathrm{mM} \mathrm{MgCl}_{2}$ $\mathrm{pH} 5.3$ ) and $5 \mu \mathrm{l}$ of $0.12 \mathrm{IU} / \mu \mathrm{l}$ nuclease $\mathrm{P} 1$ was added and incubated for 1 hour at $50^{\circ} \mathrm{C}$. Finally, $20 \mu \mathrm{l}$ Tris- $\mathrm{HCl}$ and $1 \mu \mathrm{l}$ of alkaline phosphatase $(1 \mathrm{IU} / \mathrm{ml})$ were added to each sample, incubated for 30 minutes at $40^{\circ} \mathrm{C}$, and samples then diluted either $1 / 10$ or $1 / 100$ for analysis by LC-MS/MS.

Sample Preparation for LC-MS/MS Assay of Cytosolic Thiopurine Metabolites. MOLT-4 cell pellets were defrosted on ice and ice-cold propanol (200 $\mu \mathrm{l})$ immediately added. Propanol was evaporated from the cells using a TurboVap LV automated evaporation system (Biotage, Hengoed, UK) with nitrogen gas ( $5 \mathrm{psi}$ ). Once completely dry, $200 \mu \mathrm{l}$ of deionized water was added, samples vortexed, and centrifuged $16,000 \mathrm{~g}$ for 10 minutes. The supernatant was transferred into a high-performance liquid chromatography (HPLC) glass insert for subsequent LC-MS/MS analysis.

LC-MS/MS and Reagents. The thiopurine metabolite standards, as listed above, were from Jena Bioscience. 6-Thio-2-deoxyguanosine standard (deoxythioguanosine; dTG) was from Carbosynth (Compton, UK). 6-(Methylthio)-9Hpurine-d3 was from Toronto Research Chemicals Inc. (Toronto, Canada). HPLC grade acetic acid was from Fisher Scientific (Loughborough, UK). Lymphoprep was from Axis-Shield and Pierce BCA kit was from Thermo Fisher Scientific (Cramlington, UK). All other reagents were from Sigma-Aldrich. Chromatographic separations were performed with a Shimadzu Prominence UFLC (Shimadzu, Kyoto, Japan).

An API4000 triple quadrupole MS/MS (Applied Biosystems, Foster City, CA) was used for analysis with electrospray ionization (ESI) performed in positive ion mode using nitrogen gas. Optimization of MS/MS parameters for all analytes was performed by selecting precursor ions and determining the most prominent four product ions. The three best were then further optimized for fragmentation and voltage parameters until the most abundant and robust product ion could be ascertained. Quantification of analytes was performed in multiple reaction monitoring (MRM) mode: mass transitions and optimized MS/MS parameters are given in Supplemental Table 1 and validation methods in Supplemental Information and Supplemental Table 2. Analyst software v1.5 (SCIEX, Framingham, MA) was used for sample analysis, peak integration and analyte quantification.

LC-MS/MS Analysis of Cytosolic Thiopurine Metabolites. Chromatographic separation of thiopurine metabolites was achieved using a Clarity OligoWAX column $(150 \times 4.6 \mathrm{~mm})$ and SecurityGuard Oligo-WAX guard column $(4 \times 3 \mathrm{~mm})$ both from Phenomenex (Cheshire, UK) maintained at $30^{\circ} \mathrm{C}$. Analytes were eluted with mobile phases of $0.01 \mathrm{M}$ aqueous ammonium acetate $\mathrm{pH} 8.0$ (A) and $0.01 \mathrm{M}$ aqueous ammonium acetate $\mathrm{pH} 10.01$ (B). The mobile phase system consisted of a starting condition at $90 \%$ A followed by a 0.5 minute gradient from $90 \%$ A to $80 \%$ A at 1.5 minutes. A 2 minute gradient from $80 \%$ A to $0 \%$ A was carried out at 3.5 minutes with those conditions maintained until 13.5 minutes when the column was returned to $10 \% \mathrm{~A}$ in a 0.5 minute gradient. The flow rate was $0.7 \mathrm{ml} / \mathrm{min}$ and a post-column flow splitter was used to divert $70 \%$ of mobile phase to waste and improve ionization. Standards and samples were injected in a volume of $50 \mu \mathrm{l}$. Standards were freshly diluted from a secondary dilution of
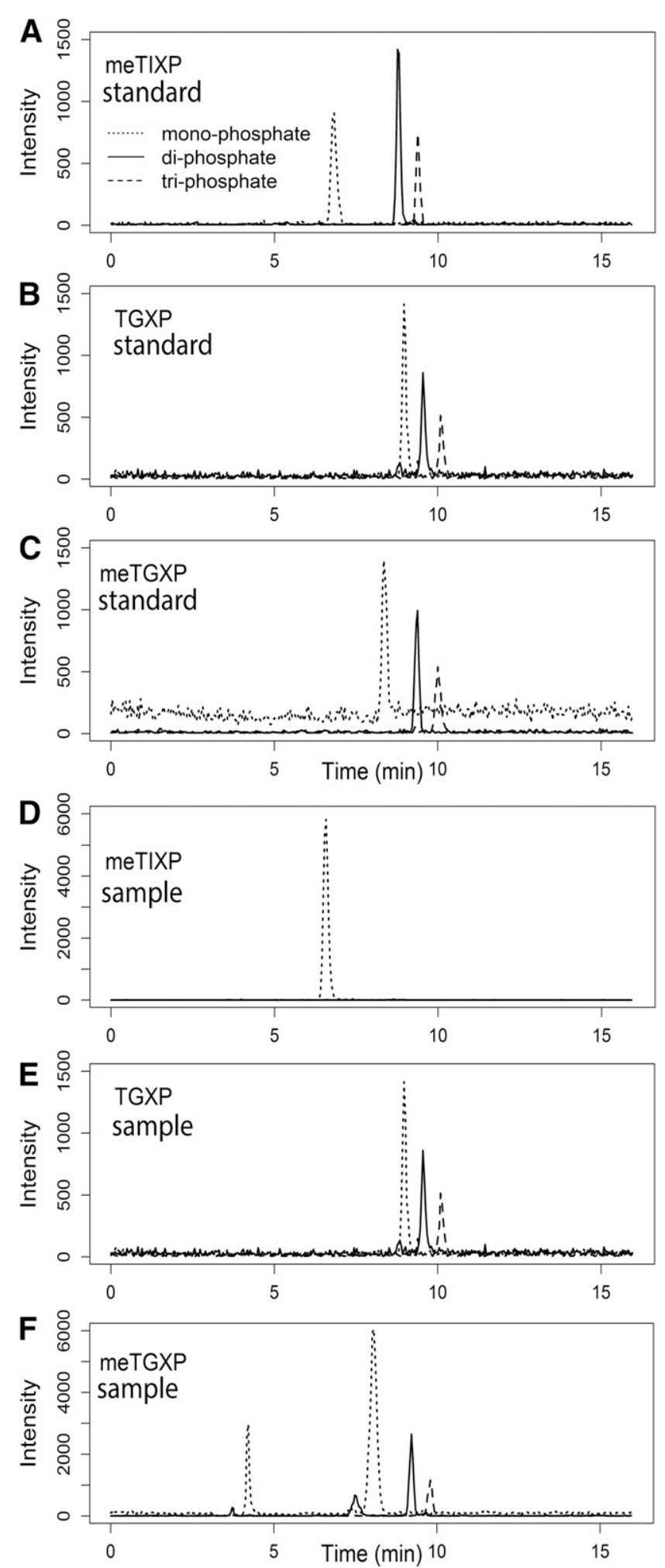

Fig. 2. LC-MS/MS profiles for thiopurine nucleoside phosphates in standard mixtures and MOLT-4 cell extracts (samples), where $\mathrm{X}$ indicates mono-, di-, or tri-phosphate. For each plot in (A-F) dotted lines represent the monophosphates, solid lines represent the diphosphates, and dashed lines represent the triphosphates [legend in (A)]. (A) Methyl-thioinosine nucleoside phosphate (meTIXP) standards; (B) thioguanosine nucleoside phosphate (TGXP) standards; (C) methylthioguanosine nucleoside phosphate (meTGXP) standards; (D) MOLT-4 cell extract (sample) analyzed for meTIXPs; (E) MOLT-4 cell extract analyzed for TGXPs; (F) MOLT-4 cell extract analyzed for meTGXPs; in this trace, the identities of additional peaks that were not eluted at the retention times of the standards are unknown. Ordinate scales are arbitrary intensity units. 

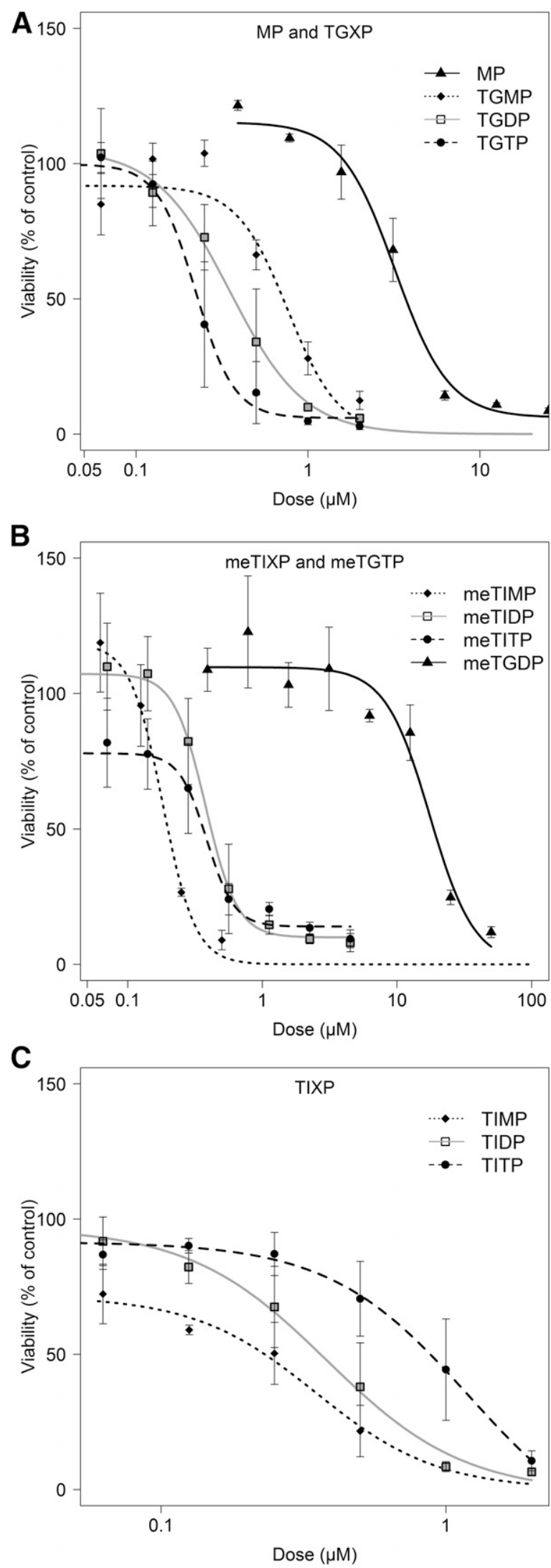

Fig. 3. Viability curves for MOLT- 4 cells treated for 72 hours with MP or thiopurine metabolites: (A) MP (solid black line, $\boldsymbol{\Lambda}$ ), TGMP (dotted black line, $\bullet$ ), TGDP (solid gray line, $\square$, gray fill), TGTP (dashed black line, $\mathbf{0}$ ); (B) meTIMP (dotted black line, $\diamond$ ), meTIDP (solid gray line, $\square$, gray fill), meTITP (dashed black stock on the day of analysis, and the secondary dilutions were used within 6 months of preparation from the primary stocks received from the manufacturer; the standards were stable under this protocol. Optimum settings for MS/MS detection were: curtain gas, 10; ion source gas 1, 40; ion source gas 2, 50; ion spray voltage, 5500; collision gas, 6; entrance potential, 10; ionization temperature, $400^{\circ} \mathrm{C}$. Examples of LC-MS/MS profiles for standards and samples are shown in Fig. 2.

LC-MS/MS Analysis of Deoxythioguanosine in DNA. Chromatographic separation of dTG and deoxyadenosine from P1 nuclease digestion of DNA was achieved using a XSelect HSS T3 $3.5 \mu \mathrm{m} 4.6 \times 100 \mathrm{~mm}$ (Waters) XSelect HSS T3, a VanGuard Cart $3.5 \mu \mathrm{m} 3.9 \times 5 \mathrm{~mm}$ guard column maintained at $30^{\circ} \mathrm{C}$, and mobile phases of aqueous $0.05 \%$ formic acid and $0.05 \%$ formic acid in acetonitrile (Coulthard et al., 2016). Standards were prepared from stock solutions of $100 \mu \mathrm{g} / \mathrm{ml}$ deoxyadenosine and $2000 \mathrm{ng} / \mathrm{ml} \mathrm{dTG;} \mathrm{standards} \mathrm{and} \mathrm{samples} \mathrm{were} \mathrm{injected} \mathrm{in} \mathrm{a}$ volume of $50 \mu \mathrm{l}$ (Coulthard et al., 2016). Two standard curves and three sets of controls for each metabolite were included in each assay.

In Silico Modeling. Interconversion between thioguanosine nucleoside mono-, di-, and tri-phosphates was modeled using the biochemical simulation software CoPaSi version 4.12 (Hoops et al., 2006). Rate constants, based on values in the BRENDA enzyme database (www.brenda-enzymes.org) (Placzek et al., 2017), were fixed and rate values $(\mathrm{V})$ for rate equations in the model fitted to experimental data.

Data Analysis. Viability data were obtained in three separate experiments for each compound. Data were blank (medium but no cells) corrected and expressed as a proportion of control cells treated with vehicle only. Mixed-effects models to account for random differences between experiments were used to estimate the mean and S.E. for the upper asymptote, slope and $\mathrm{EC}_{50}$ from three-parameter logistic curves with the "nlme" package in R version 3.1.2 (R Core Team, 2016). $\mathrm{EC}_{50}$ was defined with respect to the maximum and minimum of the logistic curves rather than the viability of untreated control cells; some of the compounds showed hormetic effects by increasing cell growth (viability) at low doses and this was reflected by fitted upper asymptotes $>100 \%$ of control. To avoid over-fitting, the lower asymptote was fixed at zero for data where viability was approaching zero but the lower asymptote was just outside the data range, but otherwise was fixed on estimates obtained from a four-parameter curve fit with all data using package "drc" in R (Ritz et al., 2015). For two drugs where mixed-effects fits did not converge to a solution, four-parameter logistic curves were fitted to each experiment using $\mathrm{drc}$ and the mean of separate $\mathrm{EC}_{50}$ values calculated. Data are expressed as means \pm S.E.M. Partial correlation and principal components analyses were carried out using the R packages "ppcor" and "FactoMineR," respectively.

\section{Results}

\section{Cytotoxicity of Thiopurine Metabolites}

To assess the cytotoxicity of individual thiopurine metabolites, an extended incubation time of 72 hours, representing approximately three cell doublings, was used to assess the effects on cell viability. This timescale was also used for assessing the role of dTG incorporation into DNA as a mechanism of cytotoxicity of the parent thiopurine drug or its metabolites. After incubation with cells for 72 hours, the parent drug, $\mathrm{MP}$, had an $\mathrm{EC}_{50}$ of the order of $3 \mu \mathrm{M}$ (Fig. 3; Table 1). With the exception of the thioinosine di- and tri-phosphates and the methylated thioguanosine nucleotides, MP metabolites were more toxic, with $\mathrm{EC}_{50}$ values ranging from 0.6 to $<0.2 \mu \mathrm{M}$. The thioguanosine nucleotides varied in cytotoxicity with TGTP the most toxic $\left(\mathrm{EC}_{50} 0.265 \mu \mathrm{M}\right)$ and TGMP the least $\left(\mathrm{EC}_{50} 0.619 \mu \mathrm{M}\right)$. The methylated thioguanosine nucleotides had the lowest toxicities of all, in spite of being able to enter cells (see below), and only meTGTP elicited a cellular response, albeit at a very high concentration $\left(\mathrm{EC}_{50} 15.37 \mu \mathrm{M}\right)$. TIMP is a direct derivative

line, ๑), meTGDP (solid black line, $\mathbf{\Delta}$ ); (C) TIMP (dotted black line, $\bullet$ ), TIDP (solid gray line, $\square$, gray fill), TITP (dashed black line, $\bullet$ ). 
TABLE 1

Effects of individual metabolites on MOLT-4 cell viability

Data are listed in order of decreasing cytotoxicity. Model fitted: ${ }^{m}$ mixed-effects; ${ }^{\S}$ mean from separate curves.

\begin{tabular}{lcc}
\hline \multicolumn{1}{c}{ Drug } & $\mathrm{EC}_{50}(\mu \mathrm{M})$ & S.E. \\
\hline meTIMP & 0.185 & $0.026^{\mathrm{m}}$ \\
TGTP & 0.265 & $0.076^{\S}$ \\
TIMP & 0.345 & $0.055^{\mathrm{m}}$ \\
TGDP & 0.360 & $0.104^{\mathrm{m}}$ \\
meTIDP & 0.393 & $0.085^{\mathrm{m}}$ \\
meTITP & 0.396 & $0.039^{\S}$ \\
TIDP & 0.412 & $0.097^{\mathrm{m}}$ \\
TGMP & 0.619 & $0.051^{\mathrm{m}}$ \\
TITP & 0.956 & $0.214^{\mathrm{m}}$ \\
MP & 2.92 & $0.415^{\mathrm{m}}$ \\
meTGTP & 15.37 & $3.556^{\mathrm{m}}$ \\
meTGDP & $>2000$ & $\mathrm{ND}$ \\
meTGMP & $>2000$ & $\mathrm{ND}$ \\
\hline
\end{tabular}

$\mathrm{ND}$, compounds not toxic and $\mathrm{EC}_{50}$ could not be estimated.

of the parent drug, 6-MP, via HPRT and had a relatively high cytotoxicity comparable to TGTP, but this was increased by methylation as meTIMP had the highest cytotoxicity of all the metabolites tested $\left(\mathrm{EC}_{50} 0.185 \mu \mathrm{M}\right.$; Table 1). Methylation also doubled the cytotoxicity of TITP but only had a small effect on cytotoxicity of TIDP (Table 1).

\section{Metabolism of Mercaptopurine Metabolites}

MP and Thioguanosine Nucleoside Phosphate Metabolites. Cells were incubated with MP or individual metabolites at their $\mathrm{EC}_{50}$ concentrations for 72 hours before LC-MS/MS analysis of intracellular metabolite levels. TIMP, TIDP, and TITP were not measured as there was no separation by LC and the major ions detected by MS/MS were the same for each compound. After incubation with MP, meTIMP was the major cellular metabolite, together with residual amounts of parent drug. Thioguanosine nucleoside phosphates were present at lower levels, with TGMP the most abundant and lower levels of TGDP and TGTP (Fig. 4). For cells incubated with thioguanosine nucleoside phosphates, treatment with either TGMP or TGDP produced similar distributions of all three thioguanosine phosphates, with TGDP predominating in both cases; the methylated equivalents had similar profiles but at levels one-fifth or lower of the unmethylated thioguanosine nucleotides (Fig. 4). Conversely, in cells incubated with TGTP, the profile of thioguanosine nucleotides differed markedly, with TGMP in greatest abundance and substantially lower levels of TGDP and the parent TGTP. Cells treated with MP had a distribution of thioguanosine nucleoside phosphates substantially biased toward TGMP similar to TGTP-treated cells, (Fig. 4, A and B).

To provide insight into potential mechanisms underlying differences in thioguanosine nucleotide profiles between cells treated with TGTP, and either TGMP or TGDP, steady-state thioguanosine nucleotide levels were modeled as a simple network of the three thioguanosine nucleotides in a closed system with enzyme activity representing guanylate kinase, nucleotide diphosphate kinase, GTPase, ATP apyrase (acting on TGTP), and guanosine diphosphatase (Fig. 4C). At steady-state, an initial model was a good fit to experimental data for cells treated with TGMP or TGDP with respect to the proportions of thioguanosine nucleotides but not for the experimental data for cells treated with TGTP. Refitting the model separately to experimental data for TGTP-treated cells showed that a 16-fold increase in guanosine diphosphatase activity would be sufficient to account for the thioguanosine nucleotide profile observed for cells treated with TGTP (Fig. 4D).

Methyl-Thioguanosine Nucleoside Phosphate Metabolites. Treatment of cells with meTGMP resulted in some demethylation to TGMP after 3 days, and some possible conversion to lower levels of meTGDP
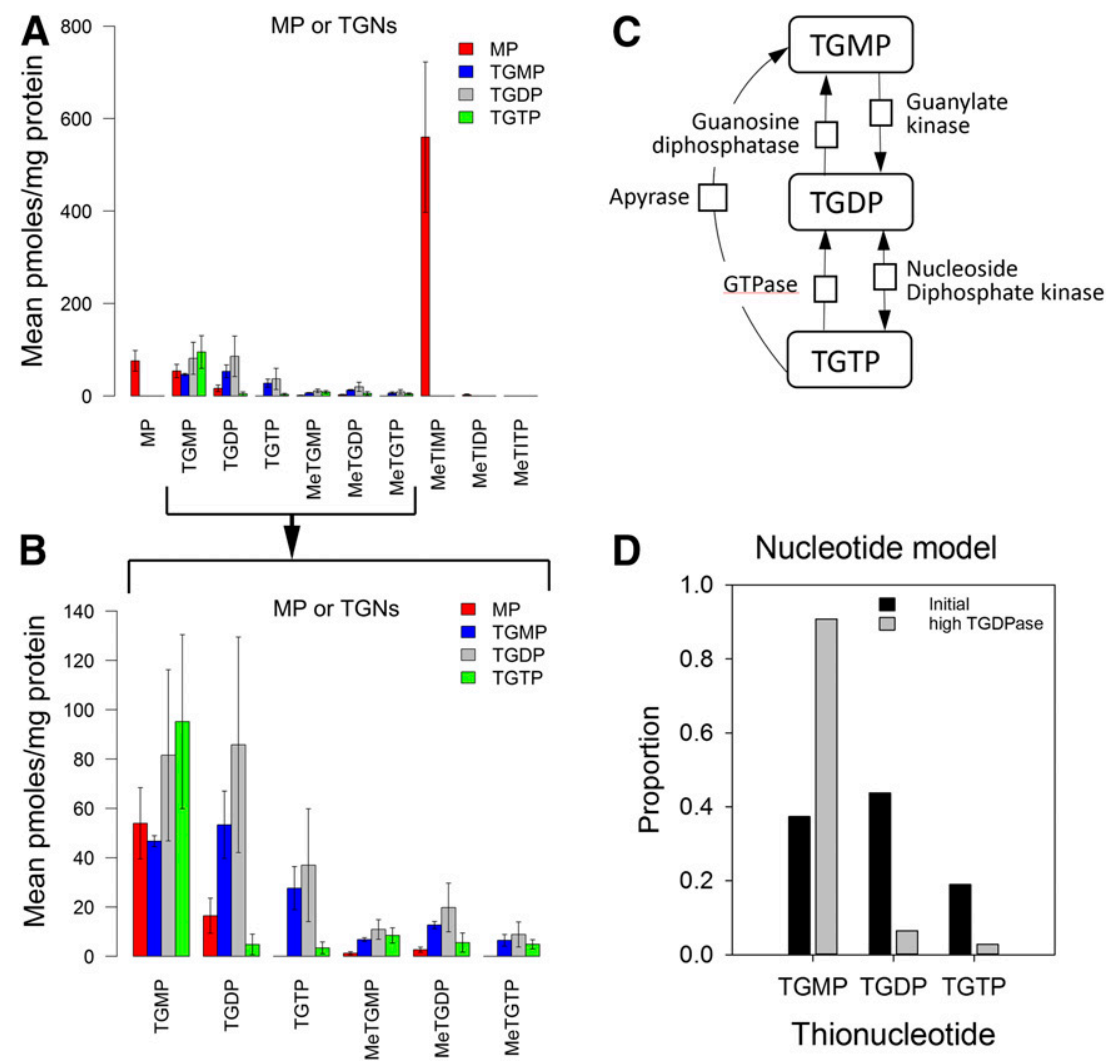

Fig. 4. Metabolites present in cells after incubation for 72 hours with MP or thiopurine nucleotides at the $\mathrm{EC}_{50}$ for cell toxicity (Table 1) and measurement by LC-MS/MS. Color is used to designate the drug used for cell treatment (specified in the legends), with bars for each drug reflecting the mean ( \pm S.E.) concentration of the drug or its metabolites extracted from the cells. (A) MOLT4 cells treated with MP or each thioguanosine nucleoside tri-phosphate; (B) data in (A) on an expanded scale to clarify intracellular thioguanosine nucleoside levels; (C) a simple nucleotide model of thioguanosine nucleoside phosphate homeostasis. $\mathrm{K}_{\mathrm{m}}$ values $(\mu \mathrm{M})$ for Henri-Michaelis-Menten irreversible kinetics were fixed at: guanylate kinase (EC 2.7.4.8: 15), GTPase (100), ATP apyrase (EC 3.6.1.5: 78), guanosine diphosphatase (EC 3.6.1.42: 100). Nucleoside diphosphate kinase activity (EC 2.7.4.6) was modeled with fixed mass action kinetics, setting $\mathrm{k}$ (forward and reverse) as 24 minutes $^{-1}$. (D) Fitting rate values (V) to data using the model in $\mathrm{C}$ gave rate values $\left(\mu \mathrm{M} \mathrm{min}^{-1}\right)$ for guanylate kinase (1578.4), GTPase (3129), ATP apyrase (15), guanosine diphosphatase (8914) and the steady-state ratios of thioguanosine nucleoside phosphates indicated by black bars. A 16-fold increase in rate values (V) for guanosine diphosphatase was sufficient to reproduce experimental data for TGTP treatment (gray bars)

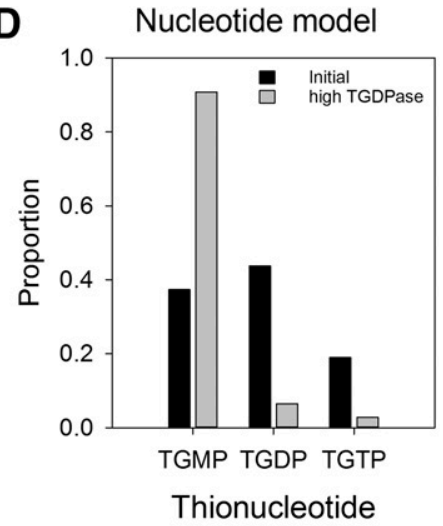



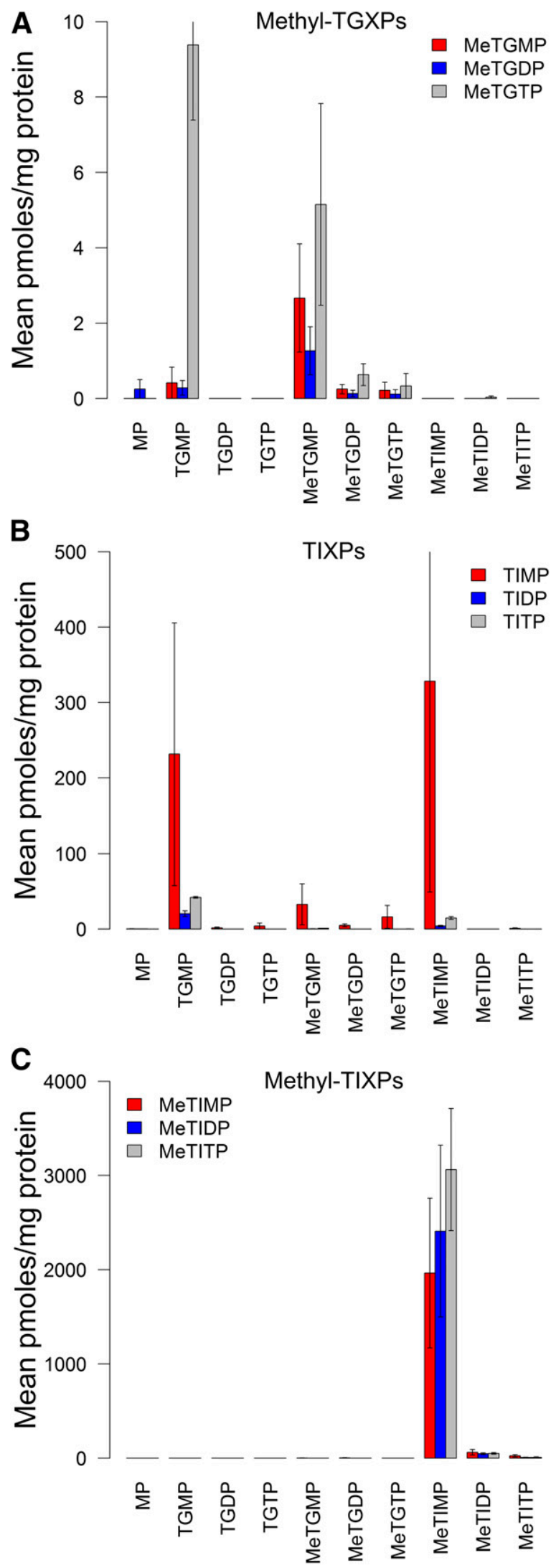

Fig. 5. Metabolites present in cells after incubation for 72 hours with thiopurine nucleoside phosphates at the $\mathrm{EC}_{50}$ for cell toxicity (Table 1) and measurement by LC-MS/MS. Color is used to designate the drug used for cell treatment (specified in the legends), with bars for each drug reflecting the mean ( \pm S.E.) concentration of each drug metabolite extracted from the cells. (A) MOLT4 cells treated with methyl-thioguanosine and meTGTP, giving a distribution similar to the unmethylated thioguanosine nucleotides in Fig. 4, A and B. Conversely, meTGDP was converted mainly to meTGMP. For cells treated with meTGTP, there was substantial dephosphorylation to meTGMP and demethylation to TGMP with TGMP the main metabolite remaining (Fig. 5A), and a similar distribution of methylated thioguanosine nucleoside phosphates to the unmethylated equivalents (Fig. 4, A and B). Thus, both dephosphorylation and demethylation reactions are involved in the metabolism of the methylated thioguanosine nucleoside phosphates.

Thioinosine and Methyl-Thioinosine Nucleoside Phosphate Metabolites. After incubation with TIMP, there was substantial methylation to meTIMP; TIMP was also metabolized to TGMP and low levels of methylated thioguanosine nucleotides were also produced (Fig. 5B), as would be predicted from known biochemical pathways. For TIDP and TITP, in contrast to TIMP, there was very little conversion to meTIMP and only very small amounts of TGMP were detectable (Fig. 5B).

MeTIMP was the major metabolite after incubation with meTIMP, meTITP, or meTIDP with no other metabolites detectable other than low levels of residual treatment metabolite (Fig. 5C). The presence of low levels of meTIDP and meTITP after treatment with meTIMP may represent low-level contaminants of $\leq 3 \%$ in the commercial source of meTIMP used; these compounds were not detectable above background at the concentrations of meTIMP analytical standards used. The results show that meTIDP and meTITP were efficiently dephosphorylated to meTIMP but without evidence for demethylation. Although meTIMP was the most toxic metabolite in viability assays, there was no correlation between metabolite $\mathrm{EC}_{50}$ in viability assays (Table 1) and the levels of meTIMP produced from each metabolite in treated cells (Spearman rank correlation, $P=0.2873$ ).

\section{Incorporation of Thioguanosine Nucleotides into DNA}

In separate experiments, MOLT4 cells were treated with parent drug or each of the metabolites (except TIDP and TITP; Fig. 6) at EC $_{50}$ concentrations for 72 hours and DNA extracted for assay of dTG. DNA-incorporated dTG was detectable significantly above background (ANOVA, Dunnett post-hoc test, $P<0.05$; Fig. 6) only in cells treated with MP $(P<0.001)$, TGDP $(P<0.001)$, TGTP $(P=0.03)$, or TIMP $(P=0.006)$. There was no significant correlation between $\mathrm{dTG}^{\text {DNA }}$ and metabolite $\mathrm{EC}_{50}$ in viability assays (Spearman rank correlation, $P=0.3614)$. Apart from the low $\mathrm{dTG}^{\mathrm{DNA}}$ after TGMP treatment, these results confirm the expected route of incorporation of thioguanosine metabolites of MP into DNA via TIMP, TGDP, and TGTP, and show that meTIMP cytotoxicity was independent of $\mathrm{dTG}^{\mathrm{DNA}}$.

\section{Data Integration}

The data for cytotoxicity $\left(\mathrm{EC}_{50}\right)$ to different metabolites, $\mathrm{dTG}^{\mathrm{DNA}}$, and metabolite levels after treatment of cells with different metabolites for 72 hours were summarized using partial correlation and principalcomponents analyses. To reduce the number of variables, measurements of mono-, di-, and tri-phosphates were combined to give thioguanosine nucleoside phosphates (TGXP), methyl-thioguanosine nucleoside phosphates (meTGXP), and methyl-thioinosine nucleoside phosphates (meTIXP). There was a significant partial correlation between TGXP

nucleoside phosphates (methyl-TGXPs,); (B) MOLT4 cells treated with thioinosine nucleoside phosphates (TIXPs, where X represents mono-, di-, or tri-phosphate); (C) MOLT4 cells treated with methyl-thioinosine nucleoside phosphates (methyl-TIXPs). 


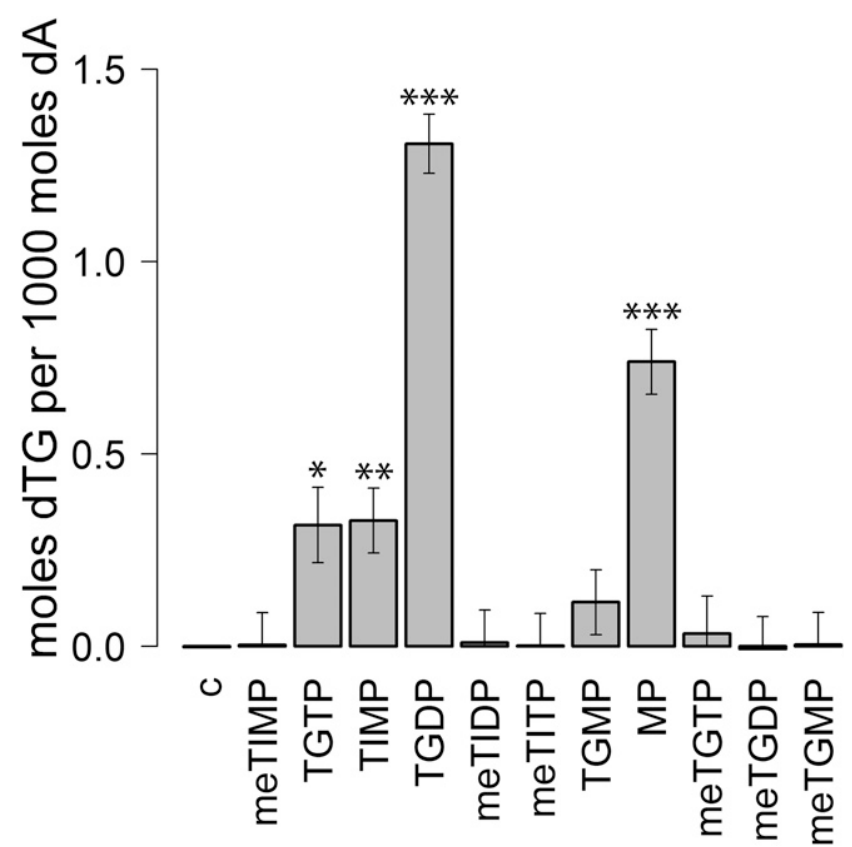

Fig. 6. Levels of deoxythioguanosine (dTG) incorporated into DNA (moles dTG per 1000 moles of deoxyadenosine [dA]) of MOLT4 cells after culture with equitoxic doses of thiopurine metabolites. Metabolites were used at their $\mathrm{EC}_{50}$ concentrations (Table 1) and the cells cultured for 72 hours before extraction and analysis. Bars are arranged, left to right, in order of decreasing cytotoxicity as given in Table 1. TIDP and TITP were not included in these experiments as they would be expected to contribute only by dephosphorylation to TIMP. Values were compared by ANOVA to the control (MOLT4 cells without thiopurine; c) with Dunnett posthoc test; error bars were derived from ANOVA and statistical significance compared with the background control (no thiopurine treatment) is indicated by: ${ }^{*} P<0.05$; $* * P<0.01 ; * * * P<0.001$.

and meTGXP $(P=0.005)$, as might be predicted; the partial correlation between dTG $^{\text {DNA }}$ and TGXP approached significance $(P=0.065)$ and such a relationship would also be expected. However, there were no significant partial correlations between $\mathrm{EC}_{50}$ and either $\mathrm{dTG}^{\mathrm{DNA}}$ or thioguanosine metabolites $(P>0.1)$. In a principal component analysis of $\mathrm{dTG}^{\mathrm{DNA}}$ and thioguanosine metabolites as independent variables, $74 \%$ and $18 \%$ of the variance was accounted for by two principal components (PC1 and PC2, respectively; Supplemental Table 3). PC1 had major contributions from dTG ${ }^{\mathrm{DNA}}$, TGXP, and meTGXP, whereas meTIXP was the dominant component $(82.7 \%$ ) of PC2 (Table 2$)$, with a small contribution from dTG ${ }^{\mathrm{DNA}}(13.6 \%)$. There was no significant correlation of $\mathrm{EC}_{50}$ with $\mathrm{PC} 1(P>0.5$; Pearson correlation coefficient $=$ $\left.-0.24 ; \mathrm{t}_{8}=-0.66\right)$, but the correlation between $\mathrm{EC}_{50}$ and $\mathrm{PC} 2$ approached significance $(P=0.059$; Pearson correlation coefficient $=$ $\left.-0.62 ; \mathrm{t}_{8}=-2.21\right)$, suggesting that methyl-thioinosine nucleoside phosphates might be key drivers of cell toxicity.

\section{Discussion}

Although in the past many studies of the biochemistry of thiopurines have focused on red blood cells, such studies will not capture the complexity of biologic responses to MP in nucleated cells, the biologic targets for thiopurine therapy (Duley and Florin, 2005). Reliable measurement of thiopurine metabolites in isolated blast cells from leukemic patients is notoriously difficult as exemplified by the paucity of published methods. A relevant in vitro model is important for the development of appropriate analytical methods for elucidating the contribution of different thiopurine metabolites to cytotoxicity and the mechanism of action of MP. In this respect, the present study is
TABLE 2

Contributions (\%) of variables to principal components (PC)

\begin{tabular}{lcccc}
\hline Variable & PC1 & PC2 & PC3 & PC4 \\
\hline DNA & 24.9 & 13.6 & 56.77 & 4.7 \\
TGXP & 32.02 & 2.9 & 5.55 & 59.5 \\
meTGXP & 30.05 & 0.76 & 33.43 & 35.76 \\
meTIXP & 13.04 & 82.7 & 4.25 & 1.97 \\
\hline
\end{tabular}

meTIXP, methyl-thioinosine nucleoside phosphates.

unique in assessing how thiopurine cytotoxicity and incorporation of fraudulent bases into DNA is related to the levels of different thiopurine metabolites.

The results emphasize that the cytotoxicity of thiopurines on nucleated cells will be combinatorial effects of different mechanisms. The incorporation of thioguanosine nucleotides into DNA promotes cell-cycle arrest (Karran, 2006) and increases the chance of DNA strand breaks (Fairchild et al., 1986; Lennard, 1992). These effects will account for some of the cytotoxicity of MP and metabolites such as TGDP. Overall, the incorporation of dTG into DNA may be a minor mechanism of cytotoxicity in MOLT4 cells. Recent studies have failed to find a good correlation between dTG incorporation into DNA and clinical efficacy as immunosuppressants (Coulthard et al., 2016, 2017), but the converse has been established for acute lymphoblastic leukemia (Nielsen et al., 2017). However, it is important to recognize the very different contexts of disease biology between uses of thiopurines as anticancer drugs versus thiopurines as immune system modulators.

MP can exert a cytotoxic effect in cells with high levels of TPMT without measurable levels of $\mathrm{dTG}^{\mathrm{DNA}}$ (Coulthard et al., 2002), and our analyses of thiopurine metabolites after treating cells with the parent drug or its known metabolites highlight perturbations to biochemical pathways that may underlie alternative mechanisms of cytotoxicity, even for leukemia cells in vitro. LC-MS/MS data show that all metabolites were able to enter the cells, and for simplicity in interpretation we assume comparable rate constants.

MeTIMP was the most toxic metabolite in viability assays and was the major metabolite in MP-treated cells after 72 hours. There was no evidence for metabolism of meTIMP to thioguanosine nucleotides for incorporation into DNA, or evidence of demethylation to other thionucleotide metabolites. From other studies there is direct evidence that meTIMP is an inhibitor of phosphoribosyl pyrophosphate amidotransferase (Tay et al., 1969; Vogt et al., 1993) and will reduce cell viability as a consequence of the inhibition of DNPS (Coulthard et al., 2002, 2011) and decreased RNA synthesis. Inhibition of DNPS will increase incorporation of dTG into DNA as a consequence of a reduction in the endogenous nucleotide pool (Bokkerink et al., 1993; Ebbesen et al., 2013); therefore, inhibition of DNPS via meTIMP in MOLT4 cells will be a substantial factor in the cytotoxic effects of MP, necessitating reliance on the nucleotide salvage pathway to sustain DNA replication (Coulthard et al., 2011). MOLT4 cells express the enzyme methylthioadenosine phosphorylase (Yu et al., 1997), an enzyme required for the salvage of adenine and methionine, but cells in which the MTAP gene is deleted are more sensitive to MeTIMP because they lack the ability to ameliorate the effects of DNPS inhibition via nucleotide salvage (Coulthard et al., 2011). The purine salvage pathway has relatively low activity in lymphoblasts (Bokkerink et al., 1993) and meTIMP as a major MP metabolite probably plays a clinical role in reducing levels of endogenous nucleotides, therefore enhancing the incorporation of dTG into DNA (Hedeland et al., 2010; Ebbesen et al., 2013). MeTIMP also contributes to a decrease in DNA methyltransferase levels and global demethylation (Hogarth et al., 2008). 
The amounts of meTIDP and meTITP detected as MP metabolites were very low compared with meTIMP, and were also low in cells treated with these methylthioinosine nucleotides, indicating effective dephosphorylation to meTIMP and suggesting that the in vitro cytotoxicity of these compounds resulted from conversion to meTIMP. Although it has been stated that TITP is a substrate for TPMT (Stocco et al., 2009; Seinen et al., 2013), the evidence on which this claim is based is not clear and in our study there was no indication of substantial methylation of TIDP or TITP. Bearing in mind that TIXPs were not measured, there was no evidence that meTIMP was demethylated to TIMP leading to thioguanosine nucleoside phosphate synthesis. With respect to the thioinosine nucleoside phosphates, TIMP was the most cytotoxic presumably as a result of methylation to meTIMP but with a contribution from $\mathrm{dTG}^{\mathrm{DNA}}$ via metabolism to TGMP.

In addition to meTIMP, MP was metabolized to the three thioguanosine nucleoside phosphates, with TGMP in excess over TGDP and TGTP, a pattern also produced by treating cells with TGTP but not TGDP or TGMP. A higher level of guanosine diphosphatase activity in response to TGTP or MP compared with TGDP or TGMP could account for the comparative effects of the different thioguanosine nucleoside phosphates in these experiments. However, the ratios of thioguanosine nucleotides in treated cells were markedly different from an expectation on the basis of the endogenous distribution of guanosine nucleotides in normal cells, where guanosine-tri-phosphate would normally be in a $\sim 10$-fold excess over the mono- and di-phosphates (Traut, 1994). With reference to a simple dynamic model of the three thioguanosine nucleoside phosphates (Fig. 4C), this implies a substantial reduction in guanylate kinase and nucleoside diphosphate kinase activities in MOLT4 cells as a result of MP or thioguanosine nucleotide treatment, accompanied in MP- or TGTP-treated cells by a possible induction of guanosine diphosphatase activity. Thus, changes in endogenous guanosine nucleotide homeostasis, potentially with substantial reductions in endogenous GTP levels as a result of thioguanosine nucleotide-mediated changes in enzyme activities, could be responsible for the inhibition of Rac1 signaling leading to apoptosis in mercaptopurine-treated cells (Tiede et al., 2003). This is an area of substantial importance for future investigation.

The thioguanosine nucleoside phosphates were all present as methylated and unmethylated metabolites. MeTGMP is 12-fold less potent than meTIMP as a DNPS inhibitor (Allan and Bennett, 1971), but all three methylated thioguanosine nucleotides had substantially less cytotoxicity that their unmethylated equivalents. Therefore, methylation of thioguanosine nucleoside phosphates via TPMT is an effective mechanism for reducing the toxicities of these metabolites. In contrast to the lack of evidence for demethylation of methylthioinosine nucleotides, there was clear evidence for demethylation of meTGMP. Most of the meTGDP and meTGTP ended up as either meTGMP or TGMP, consistent with effective demethylation and guanosine diphosphatase activity. There are few studies on the demethylation of meTGMP other than in the context of DNA, but in view of the in vitro evidence, this needs to be considered as a biologically relevant activity.

All the thioguanosine nucleoside phosphates resulted in $\mathrm{dTG}^{\mathrm{DNA}}$ in treated cells, although this was not significantly above background with TGMP, and was greatest with TGDP, as would be expected from the role of GDP as a precursor for dGTP synthesis for DNA replication (Traut, 1994). However, other mechanisms of cytotoxicity are clearly involved as TGTP was the most cytotoxic despite the lower dTG incorporation into DNA compared with TGDP. This greater cytotoxicity could result from greater disruption to Rac1 signaling implied by the lower levels of TGTP in TGTP-treated cells.

The lack of correlation between the cytotoxicity of different thiopurine metabolites and the meTIMP or $\mathrm{dTG}^{\mathrm{DNA}}$ produced by cellular metabolism implies that at least three mechanisms may contribute to the biologic activity of MP: meTIMP cytotoxicity by inhibition of DNPS or other cellular functions, reduction in viability as a result of dTG incorporation into DNA, and inhibition of GTP signaling by changes in enzymes important for GTP homeostasis. This is supported by the principal components analysis, which highlights the strong contribution of methylthioinosine nucleoside phosphates to cytotoxicity in combination with $\mathrm{dTG}^{\mathrm{DNA}}$ in MOLT4 cells. With respect to the parent drug, cell-, tissue-, and individualspecific variations in TPMT expression and activity, dependence on DNPS, the status of GTP homeostasis, DNA repair capacity, and the rates of drug and metabolite efflux (Wielinga et al., 2002) will all affect the contributions of different mechanisms to biologic effects. These mechanisms are amenable, with the availability of sufficient biochemical information, to modeling individually with integration into cell behavior models, an approach which will facilitate improvements in the clinical use and efficacy of thiopurine drugs. Such an approach would benefit by a deeper understanding of the biochemical pathways of thiopurine metabolism. This study emphasizes the importance of enzymes of nucleotide homeostasis, methylation, and demethylation in those pathways and will facilitate the development of dynamic biochemical models of thiopurine biochemistry.

\section{Acknowledgments}

The authors thank BBSRC for a studentship, and Newcastle Healthcare Charity, the JGW Patterson Foundation, and the MRC (Confidence in Concept award) for their support of this study.

\section{Authorship Contributions}

Participated in research design: Coulthard, Redfern.

Conducted experiments: Coulthard, McGarrity, Sahota, Berry.

Contributed new reagents or analytic tools: Berry, Coulthard, McGarrity.

Performed data analysis: Coulthard, McGarrity, Sahota, Berry, Redfern.

Wrote or contributed to the writing of the manuscript: Coulthard, McGarrity, Sahota, Berry, Redfern.

\section{References}

Allan PW and Bennett LL, Jr (1971) 6-Methylthioguanylic acid, a metabolite of 6-thioguanine. Biochem Pharmacol 20:847-852.

Bökkerink JP, Stet EH, De Abreu RA, Damen FJ, Hulscher TW, Bakker MA, and van Baal JA (1993) 6-Mercaptopurine: cytotoxicity and biochemical pharmacology in human malignant T-lymphoblasts. Biochem Pharmacol 45:1455-1463.

Coulthard S (2012) Mechanism of thiopurine action. Assoc Med Bras 58:18-23.Coulthard SA Berry P, McGarrity S, Ansari A, and Redfern CPF (2016) Liquid chromatography-mass spectrometry for measuring deoxythioguanosine in DNA from thiopurine-treated patients. $J$ Chromatogr B Analyt Technol Biomed Life Sci 1028:175-180.

Coulthard SA, Berry P, McGarrity S, McLaughlin S, Ansari A, and Redfern CPF (2017) Azathioprine with allopurinol: lower deoxythioguanosine in DNA and transcriptome changes indicate mechanistic differences to azathioprine alone. Inflamm Bowel Dis 23:946-955.

Coulthard S and Hogarth L (2005) The thiopurines: an update. Invest New Drugs 23:523-532.

Coulthard SA, Hogarth LA, Little M, Matheson EC, Redfern CPF, Minto L, and Hall AG (2002) The effect of thiopurine methyltransferase expression on sensitivity to thiopurine drugs. Mol Pharmacol 62:102-109.

Coulthard SA, Redfern CPF, Vikingsson S, Lindqvist-Appell M, Skoglund K, Jakobsen-Falk I, Hall AG, Taylor GA, and Hogarth LA (2011) Increased sensitivity to thiopurines in methylthioadenosine phosphorylase-deleted cancers. Mol Cancer Ther 10:495-504.

Dervieux T, Blanco JG, Krynetski EY, Vanin EF, Roussel MF, and Relling MV (2001) Differing contribution of thiopurine methyltransferase to mercaptopurine versus thioguanine effects in human leukemic cells. Cancer Res 61:5810-5816.

Duley JA and Florin THJ (2005) Thiopurine therapies: problems, complexities, and progress with monitoring thioguanine nucleotides. Ther Drug Monit 27:647-654.

Ebbesen MS, Nersting J, Jacobsen JH, Frandsen TL, Vettenranta K, Abramsson J, Wesenberg F, and Schmiegelow K (2013) Incorporation of 6-thioguanine nucleotides into DNA during maintenance therapy of childhood acute lymphoblastic leukemia-the influence of thiopurine methyltransferase genotypes. J Clin Pharmacol 53:670-674.

Estlin EJ (2001) Continuing therapy for childhood acute lymphoblastic leukaemia: clinical and cellular pharmacology of methotrexate, 6-mercaptopurine and 6-thioguanine. Cancer Treat Rev 27:351-363.

Fairchild CR, Maybaum J, and Kennedy KA (1986) Concurrent unilateral chromatid damage and DNA strand breakage in response to 6-thioguanine treatment. Biochem Pharmacol 35: 3533-3541.

Fotoohi AK, Coulthard SA, and Albertioni F (2010) Thiopurines: factors influencing toxicity and response. Biochem Pharmacol 79:1211-1220. 
Hedeland RL, Hvidt K, Nersting J, Rosthøj S, Dalhoff K, Lausen B, and Schmiegelow K (2010) DNA incorporation of 6-thioguanine nucleotides during maintenance therapy of childhood acute lymphoblastic leukaemia and non-Hodgkin lymphoma. Cancer Chemother Pharmacol 66: 485-491.

Hogarth LA, Redfern CPF, Teodoridis JM, Hall AG, Anderson H, Case MC, and Coulthard SA (2008) The effect of thiopurine drugs on DNA methylation in relation to TPMT expression. Biochem Pharmacol 76:1024-1035.

Hoops S, Sahle S, Gauges R, Lee C, Pahle J, Simus N, Singhal M, Xu L, Mendes P, and Kummer U (2006) COPASI-a COmplex PAthway SImulator. Bioinformatics 22:3067-3074.

Karran P (2006) Thiopurines, DNA damage, DNA repair and therapy-related cancer. Br Med Bull 79-80:153-170.

Lennard L (1992) The clinical pharmacology of 6-mercaptopurine. Eur J Clin Pharmacol 43 329-339.

Maybaum J and Mandel HG (1981) Differential chromatid damage induced by 6-thioguanine in CHO cells. Exp Cell Res 135:465-468.

Maybaum J and Mandel HG (1983) Unilateral chromatid damage: a new basis for 6-thioguanine cytotoxicity. Cancer Res 43:3852-3856.

Nielsen SN, Grell K, Nersting J, Abrahamsson J, Lund B, Kanerva J, Jónsson OG, Vaitkeviciene G, Pruunsild K, Hjalgrim LL, et al. (2017) DNA-thioguanine nucleotide concentration and relapse-free survival during maintenance therapy of childhood acute lymphoblastic leukaemia (NOPHO ALL2008): a prospective substudy of a phase 3 trial. Lancet Oncol 18:515-524

Pan BF and Nelson JA (1990) Characterization of the DNA damage in 6-thioguanine-treated cells. Biochem Pharmacol 40:1063-1069.

Placzek S, Schomburg I, Chang A, Jeske L, Ulbrich M, Tillack J, and Schomburg D (2017) BRENDA in 2017: new perspectives and new tools in BRENDA. Nucleic Acids Res 45 (D1): D380-D388.

R Core Team (2016) R: A Language and Environment for Statistical Computing. R Foundation for Statistical Computing, Vienna, Austria.

Ritz C, Baty F, Streibig JC, and Gerhard D (2015) Dose-response analysis using R. PLoS One 10 e0146021.

Seinen ML, van Asseldonk DP, de Boer NKH, Losekoot N, Smid K, Mulder CJJ, Bouma G, Peter GJ, and van Bodegraven AA (2013) The effect of allopurinol and low-dose thiopurine combination therapy on the activity of three pivotal thiopurine metabolizing enzymes: results from a prospective pharmacological study. J Crohn's Colitis 7:812-819.
Stocco G, Cheok MH, Crews KR, Dervieux T, French D, Pei D, Yang W, Cheng C, Pui CH, Relling MV, et al. (2009) Genetic polymorphism of inosine triphosphate pyrophosphatase is a determinant of mercaptopurine metabolism and toxicity during treatment for acute lymphoblastic leukemia. Clin Pharmacol Ther 85:164-172.

Swann PF, Waters TR, Moulton DC, Xu YZ, Zheng Q, Edwards M, and Mace R (1996) Role of postreplicative DNA mismatch repair in the cytotoxic action of thioguanine. Science 273 1109-1111.

Tay BS, Lilley RM, Murray AW, and Atkinson MR (1969) Inhibition of phosphoribosyl pyrophosphate amidotransferase from Ehrlich ascites-tumour cells by thiopurine nucleotides. Biochem Pharmacol 18:936-938.

Tiede I, Fritz G, Strand S, Poppe D, Dvorsky R, Strand D, Lehr HA, Wirtz S, Becker C, Atreya R, et al. (2003) CD28-dependent Rac1 activation is the molecular target of azathioprine in primary human CD4+ T lymphocytes. J Clin Invest 111:1133-1145.

Traut TW (1994) Physiological concentrations of purines and pyrimidines. Mol Cell Biochem 140:1-22.

Vogt MH, Stet EH, De Abreu RA, Bökkerink JP, Lambooy LH, and Trijbels FJ (1993) The importance of methylthio-IMP for methylmercaptopurine ribonucleoside (Me-MPR) cytotoxicity in Molt F4 human malignant T-lymphoblasts. Biochim Biophys Acta 1181:189-194.

Warren DJ, Andersen A, and Slørdal L (1995) Quantitation of 6-thioguanine residues in peripheral blood leukocyte DNA obtained from patients receiving 6-mercaptopurine-based maintenance therapy. Cancer Res 55:1670-1674.

Wielinga PR, Reid G, Challa EE, van der Heijden I, van Deemter L, de Haas M, Mol C, Kuil AJ, Groeneveld E, Schuetz JD, et al. (2002) Thiopurine metabolism and identification of the thiopurine metabolites transported by MRP4 and MRP5 overexpressed in human embryonic kidney cells. Mol Pharmacol 62:1321-1331.

Yu J, Batova A, Shao L, Carrera CJ, and Yu AL (1997) Presence of methylthioadenosine phosphorylase (MTAP) in hematopoietic stem/progenitor cells: its therapeutic implication for MTAP (-) malignancies. Clin Cancer Res 3:433-438.

Address correspondence to: Dr. Chris P. F. Redfern, Northern Institute of Cancer Research, Newcastle University, Paul O'Gorman Building, Newcastle upon Tyne NE2 4HH, UK. E-mail: chris.redfern@newcastle.ac.uk 